\title{
Development of Battery Pack Design for High Power Li-Ion Battery Pack of HEV
}

\author{
YoonCheol JEON*, GunGoo LEE*, TaeYong KIM**, SangWon BYUN**, \\ ChoongHo LEE*, KyeongBeom CHEONG*, HanYong LEE*, \\ SooSeok CHOI**, KiHo KIM**, Jun SONU**
}

\begin{abstract}
We developed the compact battery pack with structural safety and high cooling performance based on numerical simulation for hybrid electric vehicle (HEV) applications. The most important requirement in HEV battery pack is high specific power $(\mathrm{kW} / \mathrm{kg})$, which makes lightweight design of battery pack essential. By applying optimization methodology combined with structural finite element analysis, robust and lightweight module frame, which binds dozens of batteries together, was designed to minimize the deformation of batteries in case of swelling or explosion. Efficient cooling system was also achieved through the Pugh decision matrix with computational fluid dynamic analysis, to have a uniform temperature distribution and a minimized pressure drop within the pack under normal charging/discharging conditions. By employing module frame and cooling system based on numerical simulation, the compact design of lithium-ion battery pack was obtained successfully to have structural safety and cooling performance. Experiments were implemented to further validate numerical simulation of thus obtained optimal battery pack design for HEV, and they were found to be in good agreement.
\end{abstract}

\section{Keywords: Lithium Ion Battery Pack, Cooling System, Finite Element Analysis, Computational Fluid Dynamics}

\section{INTRODUCTION}

Recently, a hybrid electric vehicle (HEV) that utilizes an internal combustion engine and an electrical motor has been drawing lots of attention from both environmental and economical points of view and the development of HEV has accordingly been getting intensive [1]. As seen in Fig. 1, it is forecasted that HEV will replace the position of conventional automotive with combustion engine only in the near future [2].

One of the most important components of HEV is a rechargeable battery used for powering and regenerating, and lithium-ion battery has been widely used due to its superiority to conventional lead-acid and $\mathrm{Ni}-\mathrm{MH}$ batteries in terms of power capability. Besides the high specific power, lithium-ion battery has many other significant features. The first one is the high single cell voltage of $4 \mathrm{~V}$, approximately, which is three

* CAE Team, R\&D Center, SAMSUNG SDI, 428-5 Gongse-Dong, GiHeung-Gu, YongIn 446-577, Korea, e-mail: yooncheol.jeon@samsung.com;gg.lee@samsung.com; choongho7.lee@samsung.com; kb.cheong@samsung.com ; cae.hy.lee@samsung.com

** Energy 2 Team, R\&D Center, SAMSUNG SDI, Shin-Dong, YeongTong-Gu, Suwon 444-731, Korea, e-mail: kty703@samsung.com ; sangwon.byun@samsung.com ; sooseok.choi@samsung.com; kihokiho.kim@samsung.com ; jsonu@samsung.com times that of Ni-MH and two times that of lead-acid batteries. This point is very important when trying to integrate a system with a large number of batteries. The second is the high-energy efficiency enabled by the simple and straightforward battery reaction mechanism. In other words, there is no side reaction in the lithium-ion battery. The third feature is the good controllability of electrical management, which comes from easy state of charge (SOC) detection caused by the gradual voltage change upon discharge. In summary, lithium-ion battery is seen as the most promising candidate for HEV applications [3], [4].

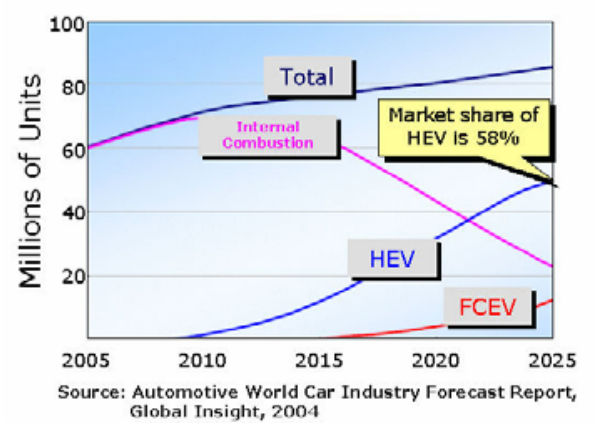

Fig. 1 Market trend of Hybrid Electric Vehicle [2]

Opposite to the above mentioned advantages, lithium-ion battery has drawbacks that excessive charging and discharging processes lead to too active 
chemical reaction at high temperatures and can cause swelling or explosion of the battery. Swelling of the battery pack may affect the safety of passengers seriously. Furthermore, non-uniform temperature distribution in the battery pack may result in premature failure of each cell. Failure may take the form of thermal runaway or acceleration in capacity fading [5-8].

In this paper, we focused on the design of compact battery pack with desired structural safety and high cooling performance. The height of pack was reduced to be installed under the seat, and the weight of pack is minimized by applying optimization methodology with finite element analysis (FEA). Efficient cooling system was also achieved through computational fluid dynamic (CFD) analysis, to have uniform temperature distribution and minimized pressure drop within the pack under normal charging and discharging conditions. By employing module frame and cooling system based on numerical simulation, a prototype of compact battery pack was manufactured. Experiments were also carried out on this optimized prototype of battery pack to further validate numerical simulation.

\section{NUMERICAL DESIGN}

\subsection{Design of Endplate}

The primary function of endplates is to constrain the batteries and barriers together with restraint rods. Endplates may bend considerably when simultaneous swelling of each battery takes place. Therefore, it is important to design the endplate having high bending strength against the direction where the bending moment is applied.

In order to enhance the bending strength and reduce its weight, corrugated endplate was developed as shown in Fig. 2. The significant feature of this structure is its high strength-to-weight ratio. The corrugated domain enables the whole structure to act as a single thick plate as a virtue of its bending strength [11-13]. The second feature is machinability due to its shape characteristics. Manufacturing cost and time will be cut down because the corrugated domain can be machined by pressing or die-casting instead of cutting.

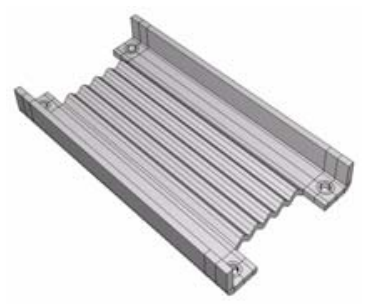

Fig. 2 Corrugated Endplate

The corrugated endplate was optimized for minimum weight by applying optimum design algorithm [14]. With the modified endplate design, approximately $20 \%$ of weight reduction in the endplate and restraint rod was obtained through optimization procedure while keeping stress level [15].

\subsection{Design of Barrier}

In a battery pack, the barriers are located between the batteries and their primary function is to make a room for cooling air to pass through them. Only elastic deformation is allowed even when excessive swelling pressure is applied to barriers, and flow loss of cooling air during operation, such as pressure drop, is to be minimized. Channel type barrier as shown in Fig. 3(a) had a drawback of high pressure drop. As an alternative, we developed bump type barrier as shown in Fig. 3(b) in order to reduce the pressure drop while keeping its structural stress level [16].

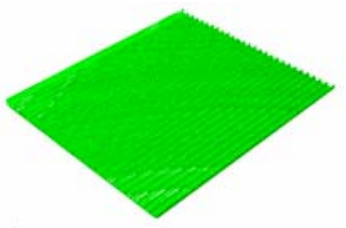

(a)

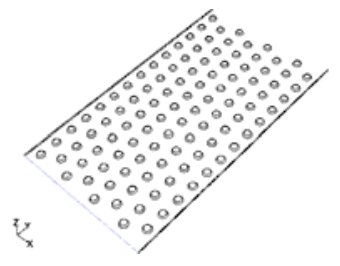

(b)
Fig. 3 Shape comparison of cooling barriers: (a) Channel type barrier (b) Bump type barrier

In Fig. 4, comparisons of structural safety and thermo-fluid characteristics for channel type and bump type barriers are performed through FEA and CFD analysis. By applying the same loading and boundary conditions, stress, pressure drop and temperature are obtained. For channel type barrier, maximum stress, pressure drop and maximum temperature are $73.2 \mathrm{MPa}$, $114 \mathrm{~Pa}$ and $308.9 \mathrm{~K}$, respectively, whereas those are $34.3 \mathrm{MPa}, 85.9 \mathrm{~Pa}$ and $309.7 \mathrm{~K}$ for bump type barrier, respectively. From the comparisons, considerable reduction in stress and pressure drop can be obtained while keeping similar temperature distribution if bump type barrier is adopted.

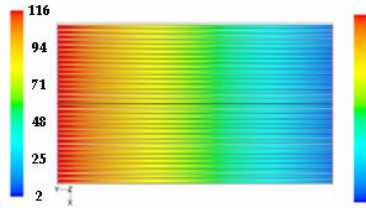

(a)

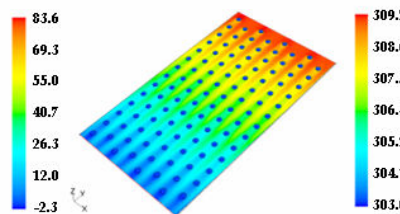

(c)

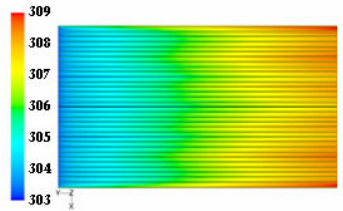

(b)

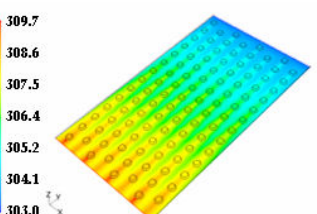

(d)
Fig. 4 Comparisons of pressure drop and temperature distribution for different barrier design; (a) and (b) are for channel type barrier and (c) and (d) are for bump type barrier 


\subsection{Design of Flow Channel}

During charging and discharging of a battery, some of energy is dissipated into the form of thermal energy, and it is implemented with CFD analysis. The ratio of thermal dissipation energy over total electrical energy defined as energy conversion rate can be obtained through experiment. Surface temperature of the battery was measured by using infrared camera (ThermaCAM ${ }^{\circledR}$ SC 2000, FLIR Systems, USA) after 200 cycles of repetitive charging and discharging. In parallel, CFD analysis using FLUENT [17] with the same boundary condition was also performed to find the energy conversion rate of the battery by comparing the temperature at specific positions between simulation and experiment. As a result, the variation of the maximum temperature and energy conversion rate with respect to ambient temperature was obtained as shown in Fig. 5.

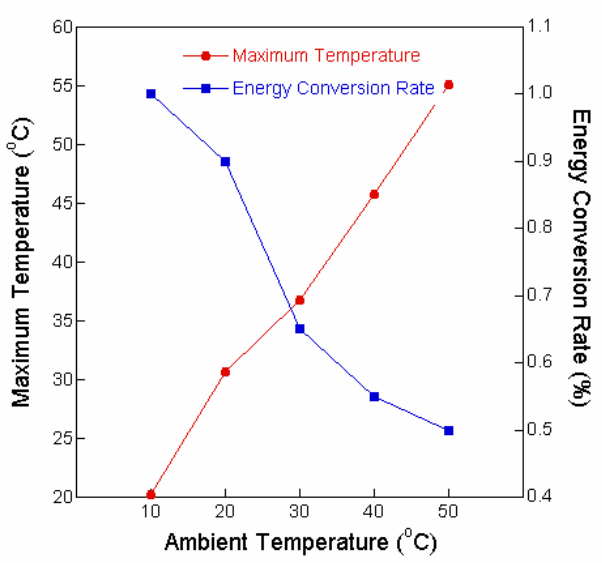

Fig. 5 Relationship of the maximum temperature and energy conversion rate with respect to ambient temperature

The criteria in the design of cooling system are as follows; (1) to minimize the static pressure drop between the inlet and outlet of the pack, (2) to keep the temperature difference between adjacent batteries within $5 \mathrm{~K}$, (3) to maintain the temperature rise inside the battery below $323 \mathrm{~K}$.

There would be many feasible configurations for the flow channel depending on how the batteries are stacked, but it should be the one to satisfy all those previously mentioned constraints on the temperature and pressure, to have low battery pack height and to be easily manufactured. In this paper, the optimal flow channel was chosen by adopting a method for concept selection using a scoring matrix called the Pugh decision matrix as shown in Table 1. Considered parameters were evaluated as being better $(+)$, the same (S), or worse (-) for the rating and the reference for this rating is based on the experimental data. By summating the ratings, type $\mathrm{A}$ was chosen as an optimal flow channel with advantages such as lower height, higher volumetric efficiency, and better manufacturability except temperature difference in the pack.
Table 1 Pugh decision matrix for optimal flow channel

\begin{tabular}{|c|c|c|c|c|}
\hline Flow channel & Type A & Type B & Type C & Type D \\
\hline Parameters & Top View & Top View & Side View & Side View \\
\hline Maximum temperature & s & s & s & s \\
\hline $\begin{array}{l}\text { Pressure Drop } \\
\text { in battery pack }\end{array}$ & - & + & S & S \\
\hline $\begin{array}{c}\text { Temperature distribution } \\
\text { in battery pack }\end{array}$ & S & + & $\mathbf{s}$ & S \\
\hline Height of battery pack & + & + & - & - \\
\hline Volumetric efficiency & + & - & - & s \\
\hline $\begin{array}{l}\text { Degree of difficulty } \\
\text { in manufacturing }\end{array}$ & + & - & s & s \\
\hline Sum of + & 3 & 3 & 0 & 0 \\
\hline Sum of $\mathrm{S}$ & 2 & 1 & 4 & 5 \\
\hline Sum of - & 1 & 2 & 2 & 1 \\
\hline
\end{tabular}

\section{EXPERIMENTAL VERIFICATION}

\subsection{Dynamic Strain and Pressure}

Fig. 6 shows the test set of battery module consisting of batteries, barriers, endplate, and restraint rods for the measurement of strain induced by the internal gas pressure of batteries during over-charging. During over-charging of the battery with the specific charging rate, pressure gage (MS5212-BM, INTERSEMA, Switzerland) installed on the cap plate between vent plug and electrode continuously measured the internal gas pressure of battery. At the same time, strain gages (YFLA-5, Tokyo Sokki, Japan) measured dynamic strain at several points on endplate and restraint rods. Synchronized signals from pressure gage and strain gages were transmitted to personal computer by way of data acquisition system (SCXI-1000 series, National Instrument, USA).

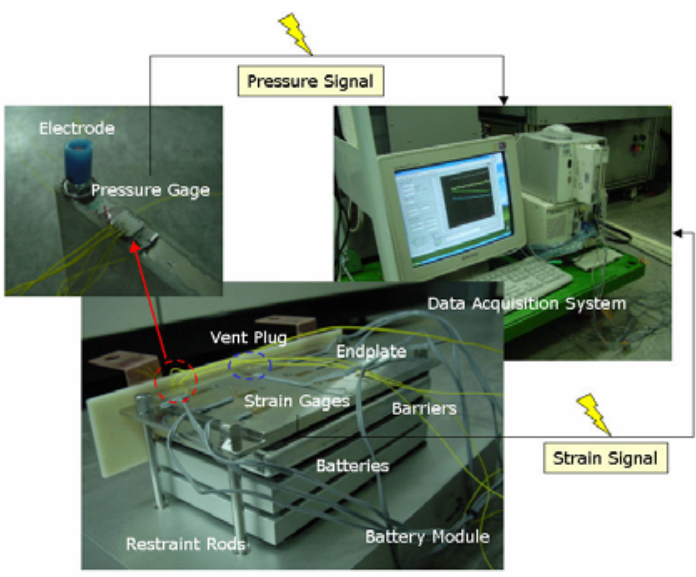

Fig. 6 Measurement system of strain and pressure of the module frame during over-charging of battery

Fig. 7 (a) shows the comparisons of strains at the endplate obtained from experiment (scattered points) and FEA (solid and dashed lines) by applying the pressure as shown in the Fig. 7 (b) at the chosen positions. When the pressure was removed completely, 
no plastic strain was found anywhere. Therefore, only elastic deformation is supposed to occur at the endplate even when the pressure inside of the battery attains to vent pressure level. From the comparison, FEA results somewhat underestimated the experimental data. However, finite element predictions mostly agreed very well with the experimental data.

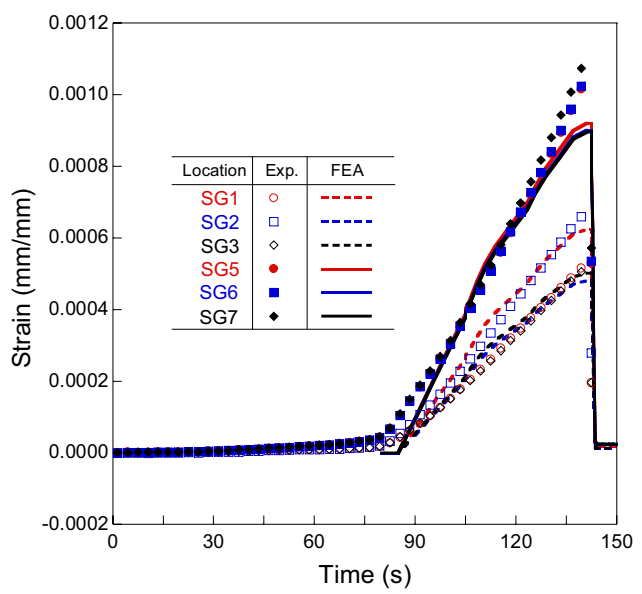

(a)

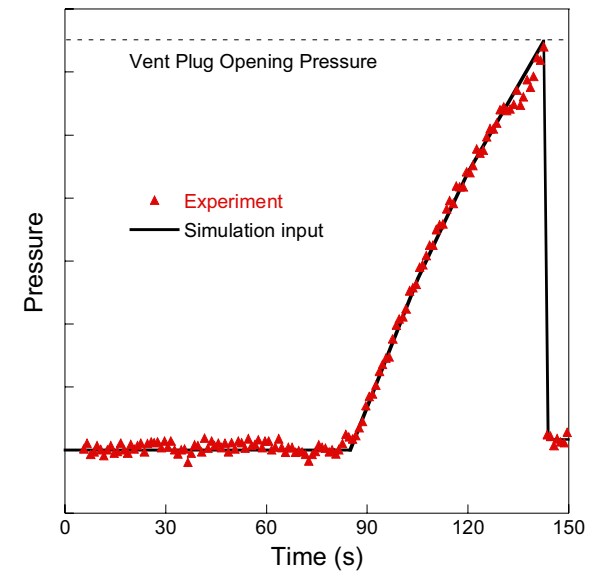

(b)

Fig. 7 (a) Comparison of strain data from experiment (scattered points) and FEA(solid and dashed lines) and (b) Measurement of pressure inside of a battery

From the response of each strain after unloading of the pressure, complete spring back occurred at both the endplate and the restraint rod, which means robustly designed module frame can prevent the subsequent deformation, which can affect the safety of passenger in a vehicle even when the simultaneous swelling in the battery takes place.

\subsection{Pressure Drop and Temperature}

In order to measure the static pressure drop across the battery pack, experimental setup was constructed as shown in Fig. 8. It is composed of flow meter, differential pressure gage, and the prototype of battery pack including cooling fan. The pressure drop across the battery pack was measured by precise differential pressure gage (MC-1006, Dwyer, USA) with resolution of $0.01 \mathrm{~Pa}$. The pressure taps were mounted on the flow walls along the upstream and downstream paths of the battery pack. The air was sucked by a variable speed fan in the flow meter and passed through the test section of the battery pack.

The static pressure drop was measured for the battery pack with barriers. From the experimental data, maximum available flow rate was $0.73 \mathrm{~m}^{3} / \mathrm{min}$ and maximum static pressure drop was $68.5 \mathrm{~Pa}$ in the battery pack. By applying the same boundary conditions, the simulation results for the maximum flow rate and static pressure drop were $0.88 \mathrm{~m}^{3} / \mathrm{min}$ and $70 \mathrm{~Pa}$, respectively. From the comparison, pressure drop was in very good agreement, however, slight difference was found in the available flow rate. Probably, minute leakage in the pack due to machining tolerance can be one of the reasons why the discrepancy between experiment and simulation in flow rate exists. However, measured pressure drop also satisfied the design criterion for pressure drop.

With the life cycle pattern of SAMSUNG SDI lithium-ion battery with T-type thermocouples (FF-T-28, SENTECH, Korea) attached, the measured inlet temperature of cooling air was $302 \mathrm{~K}$. Fig. 9 shows the comparison of the steady-state temperatures with respect to the position of barrier between experiment and simulation where the position of each barrier is numbered consecutively from the front of the pack to the back. Temperatures showing saturation behavior as inset of Fig. 9 were measured from both modules in a pack to examine whether the symmetry of temperature distribution is achieved or not. Temperatures at the right and left modules show symmetric behavior judging from that the temperature difference is within $1.1 \mathrm{~K}$. And, maximum temperature difference between experiment and simulation was $1.2 \mathrm{~K}$ at the fifth barrier from the front of the pack, which was insignificant. Experimental results show that the batteries toward the front of the pack are hotter than those toward the back and this is the similar trend with the simulation results in Fig. 10. Also, the maximum temperature differences within a battery pack from experiment and simulation were $3.2 \mathrm{~K}$ and $1.0 \mathrm{~K}$, respectively. Both results satisfied the design criterion for temperature.

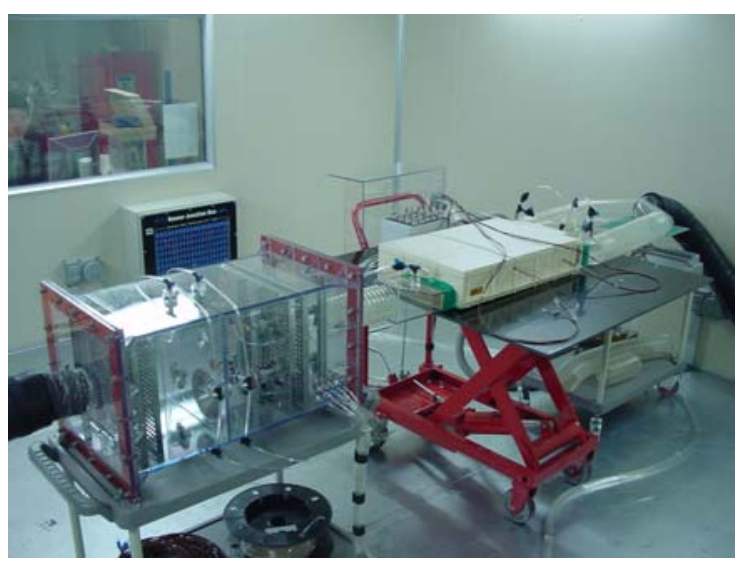

Fig. 8 Pressure drop measurement system 


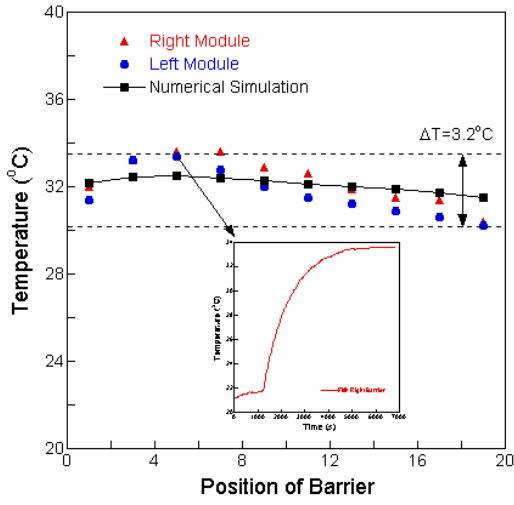

Fig. 9 Comparison of temperatures between experiment and simulation

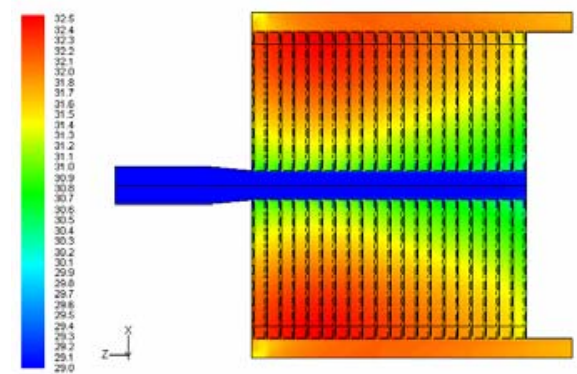

Fig. 10 Simulation results for the temperature distribution in a battery pack

From the verification of numerical design for cooling system with experiment, all the design parameters such as pressure drop, temperature difference in a pack, and maximum temperature within batteries satisfied the design criteria mentioned in section 2.2. Uniform temperature distribution with the difference less than $4 \mathrm{~K}$ in a battery pack is expected.

Table 2 Primary specifications of battery pack

\begin{tabular}{|c|c|}
\hline & SDI 2005 \\
\hline Peak Power $(\mathrm{kW})$ & 33 \\
\hline Capacity $(\mathrm{mAh})$ & 6000 \\
\hline Weight $(\mathrm{kg})$ & 26.9 \\
\hline Height $(\mathrm{mm})$ & 120 \\
\hline Volume $(\mathrm{L})$ & 27.9 \\
\hline Pressure Drop $(\mathrm{Pa})$ & 68.5 \\
\hline Max. Temperature $(\mathrm{K})$ & 306.6 \\
\hline Temp. Difference $(\mathrm{K})$ & 3.2 \\
\hline
\end{tabular}

\section{PROTOTYPE OF BATTERY PACK}

In this paper, compact design of battery pack was obtained to possess desired structural safety and high cooling performance. Considerable reduction in height, weight, and volume of pack were accomplished to enhance the specific power of pack. In Table 2, primary specifications of optimized battery pack are summarized and Fig. 11 shows the prototype of battery pack. Reduction in height enables the pack to be installed even under the seat of vehicle. In particular, reduction in volume maximizes the usage of space in the vehicle satisfying the constraint on the temperature difference within the pack.

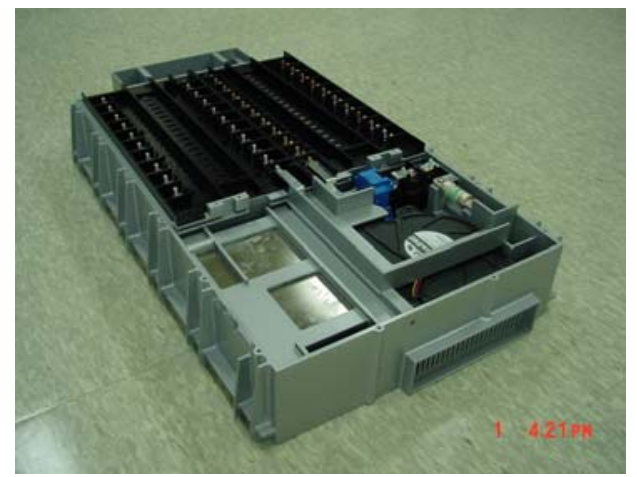

Fig. 11 Prototype of battery pack

\section{CONCLUSIONS}

We developed successfully the design of the compact battery pack with desired structural safety and high cooling performance based on numerical simulation. Durable and lightweight module frame was designed by applying optimization methodology combined with structural FEA. The experimental strain data and simulation results were found to be in good agreement. Therefore, the safety of module frame could be guaranteed only if design procedure proposed in section 2 were adopted.

Further, by applying the Pugh decision matrix with CFD analysis, efficient cooling system design was also developed. From the comparison between experimental and numerical results, their agreements were very good. By applying CFD analysis, cooling system design satisfying the fairly narrow pressure and temperature constraints can guarantee the reasonable pressure drop and the uniform temperature distribution in a battery pack.

In the next stage, we will do anti-vibration design and crash analysis for the battery pack installed in the vehicle.

\section{REFERENCES}

[1] O. Bitsche, G. Gutmann, "Systems for hybrid cars," J. Power Sources, Vol. 127, pp. 8 - 15, 2004

[2] C. Kim, Proc. Korean Society of Automotive Engineers, KSAE05-L0022, 2005.

[3] U. Kohler, J. Kumpers, M. Ullrich, "High Performance nickel-metal hydride and lithium-ion batteries," J. Power Sources, Vol. 105, pp. 139 - 144, 2002.

[4] T. Horiba et al., "Manganese-based lithium batteries for hybrid electric vehicle applications," J. Power Sources, Vol. 119, pp. 893 - 896, 2003.

[5] A. Pesaran, A. Vlahinos, and S. D. Burch, "Thermal performance of EV and HEV battery modules and packs," Proc. of the 14th Int. Electric Vehicle Symposium, Orlando, Florida 1997.

[6] H. Maleki et al., "Thermal properties of lithium ion battery and components," J. of the Electrochemical Society, Vol. 146, No. 3, pp. 947 - 954, 1999.

[7] J. Selman et al., "Cooperative research on safety fundamentals of lithium batteries," J. Power Sources, 
Vol. 97, pp. $726-732,2001$.

[8] S. Al-Hallaj, J. Prakash, J. R. Selman, "Characterization of commercial Li-ion batteries using electrochemical calorimetric measurements," J. Power Sources, Vol. 87, pp. $186-194,2000$.

[9] D. Jung et al., Proc. Korean Society of Automotive Engineers, KSAE05-L0024, 2005.

[10] T. Yaegashi, "The past, Current and Future on Hybrid Technologies," 2005 HVEC Proc. Int. Workshop on HEV, 2005.

[11] W. Chang et al., "Bending behavior of corrugated-core sandwich plates," Composite Structures, Vol. 70, pp. 81 $-89,2005$.

[12] Z. Aboura et al., "Elastic behavior of corrugated cardboard: experiments and modeling," Composite Structures, Vol. 63, pp. 53 - 62, 2004.

[13] S. Timoshenko, S. Woinowsky-Krieger, Theory of Plates and Shells, p. 367, McGraw Hill Book Co., 1959.

[14] J. Arora, Introduction to Optimum Design, p. 291, McGraw Hill Book Co., 1989.

[15] ABAQUS User's Manual, Version 6.5, ABAQUS Inc., 2005.

[16]T. Kim, Y. Jeon, and G. Lee, US Patent No. 2005-166403, 2005.

[17] Fluent User's Manual, Version 6.1, Fluent Inc., 2003.

\section{BIOGRAPHIES}

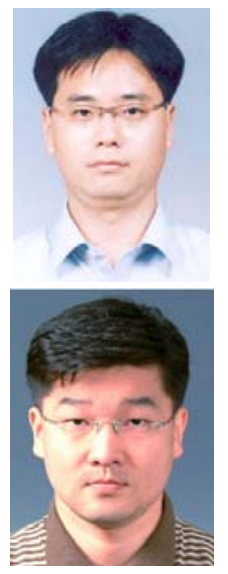

YoonCheol Jeon received the M.S. degree in Mechanical Engineering from POSTECH in 1997. $\mathrm{He}$ works as a senior engineer in $\mathrm{R} \& \mathrm{D}$ center of SAMSUNG SDI.

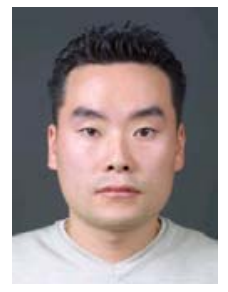

TaeYong Kim received the M.S. degree in Mechanical Engineering from Inha University in 2000. He works as an engineer in $R \& D$ center of SAMSUNG SDI.

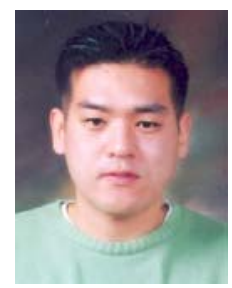

SangWon Byun received the M.S. degree in Mechanical Engineering from Inha University in 2000 . He works as an engineer in R\&D center of SAMSUNG SDI.

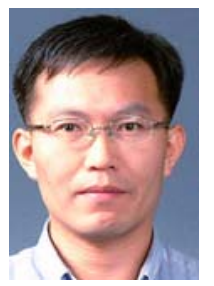

ChoongHo Lee received the Ph.D. degree in Mechanical Engineering from KAIST in 1997. He works as a senior engineer in $R \& D$ center of SAMSUNG SDI.

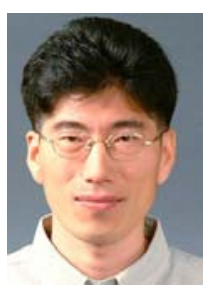

KyeongBeom Cheong received the $\mathrm{Ph}$. D. degree in Mechanical Engineering from KAIST in 1997. He works as a principal engineer in $R \& D$ center of SAMSUNG SDI.

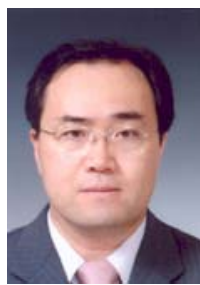

HanYong Lee received the Ph.D. degree in Applied Mathematics from KAIST in 1989. He works as a vice president in $R \& D$ center of SAMSUNG SDI and he is the head of CAE Team.

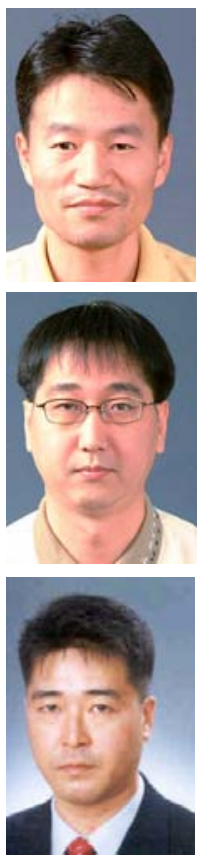

SooSeok Choi received the M.S. degree in Physics from Seoul National University in 1994. He works as a senior engineer in R\&D center of SAMSUNG SDI.

KiHo Kim received the Ph.D. degree in Materials Science and Engineering from Seoul National University in 1991. He works as a principal engineer in R\&D center of SAMSUNG SDI.

Jun Sunu received the Ph.D. degree in Metallurgy and Material Science from University of Cincinnati, USA in 1988. He works as a vice president in R\&D center of SAMSUNG SDI and he is the head of Energy 2 Team. 